\title{
Postoperative Cerebrospinal Fluid Hypovolemia in Neurosurgery
}

\section{Cheol Wan Park}

Department of Neurosurgery and Emergency Medicine, Section of Critical Care Medicine, Gil Medical Center, Gachon University, Incheon, Korea

Received: August 17, 2018

Accepted: September 19, 2018

Published: October 10, 2018

\section{Corresponding Author:}

Cheol Wan Park, M.D., Ph.D.

Department of Neurosurgery and Emergency Medicine, Section of Critical Care Medicine, Cancer Center, Gil Medical Center, Gachon University, 1141-8 Guweol-Dong, Namdong-Gu, Incheon 21556, Korea

Tel: +82-32-460-3304

Fax: +82-32-460-3899

E-mail: cwpark@gilhospital.com

\begin{abstract}
Almost all neurosurgeons have concerned with postoperative uneventful recovery of their patients. However, although rare but endlessly, unexpected postoperative adverse events have occurred. Neurosurgeons, then are embarrassed and they search the causes and/or pathogenesis of such problems. Also rare, a few neurosurgeons may have been forced to litigate because of such unexpected and thus uninformed postoperative events.

As many postoperative complications, postoperative adverse events from cerebrospinal fluid (CSF) hypovolemia may have led to tough circumstances. Although the exact pathomechanisms have not been fully cleared, a CSF hypovolemia can be spontaneous or postoperative, clinically. And both the cranial and spinal surgeries can cause CSF hypovolemia. It usually have taken benign courses, and some cases may have exhibited serious outcomes. We, neurosurgeons, then have considered the possibility of CSF hypovolemia in differential diagnosis for unanticipated postoperative neurologic deterioration.
\end{abstract}

Since only the early recognition and prompt treatment may reverse those situations, the author try to settle and summarize comprehensively the postoperative adverse events due to CSF hypovolemia through the review of recently published or frequently cited reports on the postoperative CSF hypovolemia.

Keywords: Postoperative complications; Intracranial hypotension; Brain sag; Pseudohypoxic brain swelling; Remote site intracranial hemorrhage; Cerebrospinal fluid
Cerebrospinal fluid (CSF) hypovolemia may occur spontaneously or after neurosurgical procedures. A spontaneous form of CSF hypovolemia have also known as spontaneous intracranial hypotension and usually take chronic and/or benign clinical course $^{8)}$. The other form of CSF hypovolemia is postoperative and often show problematic acute exacerbations during recovery from anesthesia or critical care except intraoperative therapeutic extensive CSF removal ${ }^{18)}$ in some particular conditions. The pathophysiologic mechanisms, though not clearly demonstrated, of these acute deteriorations which the most close pathomechanism have been seemed to have a considerable consensus among many interested neurosurgeons, have been described separately in a number of case reports and case series $^{1-13,15-17,19,21)}$.

A comprehensive description, however, for clinical entities of postoperative CSF hypovolemia is almost rare until lately. Also, the terminologies related to postoperative CSF hypovolemia have not been uniformly standardized yet. The 
aim of this brief review of literatures is attempt to settle and analyze postoperative CSF hypovolemia comprehensively. As a postoperative complication, both the cranial and spinal ${ }^{16)}$ surgeries, CSF hypovolemias might have been expressed in three different clinical types of phenomena; 1) brain sag, 2) pseudohypoxic brain swelling (PHBS), 3) remote site hemorrhage. Basically, these three clinical phenomena do not have any new theoretical concept. The author's proposed classification and pathomechanism for these phenomena is shown in Fig. 1.

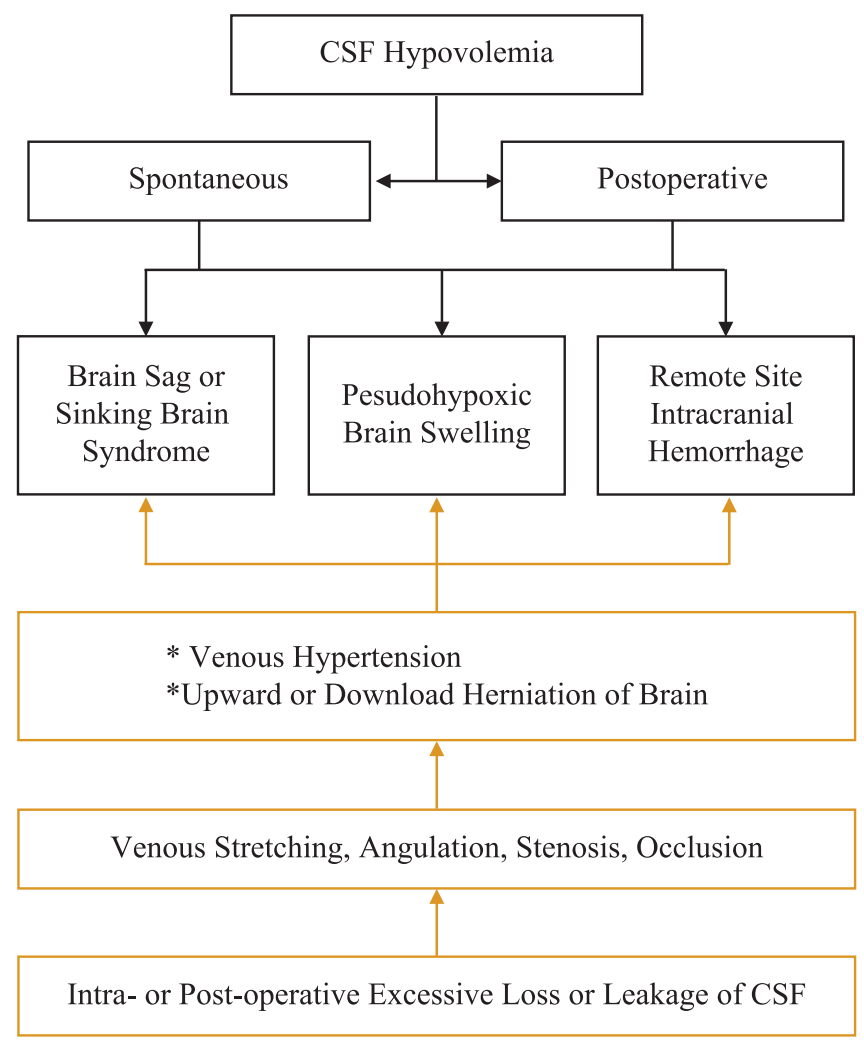

Fig. 1. A proposed pathomechanism of three clinical phenomena due to postoperative cerebrospinal fluid hypovolemia.

Above three types of clinical entities may have, essentially identical pathophysiologic mechanisms such as venous stretching, angulation, occlusion, or disturbed intracranial venous return to heart then venous hypertension, and may arise from the intra-or post-operative excessive loss of CSF via diverse routes including subarachnoid cisternal spaces, lamina terminalis, extraventricular drain, lumbar drain or puncture, subgaleal or epidural negative vacuum drain, or traumatic fractures of the skull base. And they also may have similar symptoms and signs whether benign or malignant form although their radiologic characteristics are quite distinct one another. In the management for these three clinical phenomena, the general principles are fundamentally same. That is, flat or Trendelenberg positioning of the patient and prevention of further CSF loss. Of course, detailed methods of treatment for each type of this clinical condition by the postoperative CSF hypovolemia can be exceedingly different.

The brain sagging, also called 'sinking brain syndrome ${ }^{5)}$ is typically mild, and an upright positional headache is the most common presenting symptom. Other complaints such as nausea, vomiting, vertigo and visual disturbance may be subtle. Mild symptomatic brain sagging tends to recover spontaneously without any intervention except a few cases that may need the patients flat position for some time with or without epidural blood patches. Some brain saggings, especially immediate postoperative, may develop serious symptoms and signs such as coma with abnormal pupillary reflexes ${ }^{1,5-7,15)}$. Diagnostic criteria for severe brain sagging by Komotar et al. ${ }^{10)}$ are as followings; 1) clinical signs of transtentorial herniation, 2) head computed tomography (CT) scans revealing effacement of the basal cisterns with an oblong brainstem, 3) improvement of symptoms and/or signs upon placing the patient in the Trendelenberg position. In those circumstances, early detection and identification of brain sagging then Trendelenberg positioning, with primary cause management to prevent further CSF loss is mandatory. This management policy is extremely significant to arrest progression to irreversible neurologic deficits or death and to promote whole recovery.

PHBS, relatively newly coined and defined term, may be also caused by excessive CSF loss, especially through the subgaleal or epidural negative pressure suction drain ${ }^{19)}$. And intraoperative CSF drainage may contribute to this complication. Variable degree of severity for PHBS can occur, although majority of PHBS cases are found to be grave neurologic deficits including not awakening from anesthesia, unreactive mydriasis, generalized seizure in uneventful elective neurosurgery ${ }^{19,20)}$. This prevalence of critical severity especially, before the report by Van Roost et al. ${ }^{19)}$, may be insufficient understanding of this phenomenon. Mild to moderate degree of PHBS may occur presenting abnormal mental status, signs of abnormal brainstem function, and etc, Typical imaging characteristics are diffuse brain swelling particularly involving bilateral basal ganglia and thalami on head CT or magnetic resonance images (MRI). Angiographic findings are commonly non-specific and may demonstrate only a slow circulation time by microcirculatory disturbance from diffuse brain swelling ${ }^{19,20)}$. Principles of management are almost conclusive i.e., prevention of further CSF loss, Trendelenberg positioning, reduction of increased intracranial pressure. The prognosis of PHBS is variable, and usually depends on the time of correct diagnosis and treatment, 
then may be fatal or may show complete recovery ${ }^{19)}$.

Remote site hemorrhage is the most repeatedly reported postoperative complication among three clinical phenomena due to postoperative CSF hypovolemia as mentioned earlier $^{2,4,8,12,21}$. By definition, remote site hemorrhage is bleeding at a location or locations distant from the site of original surgery ${ }^{21)}$. Although the first report was after supratentorial craniotomy it can occur after supra- or infra-tentorial surgery especially in sitting position ${ }^{3)}$, burr hole trephination ${ }^{4}$, or spinal surgery $^{20)}$ i.e., neurosurgery of almost all kinds. Its prevalence is rather wide-ranging, from 0.2 to $4.9 \%$ after different kinds of neurosurgery $y^{2-4,8,12,21)}$. Many hypotheses have been advocated and published for decades though the exact pathophysiologic cause has not been fully revealed yet. Radiologic findings are often non-specific except remote site intracranial hemorrhage in epidural, intracerebral, or cerebellar, which may appear solitary or multiple on head CT and/or MRI. Once such a adverse event develop it can become fatal. Surely, perfect restoration from neurologic deterioration after conservative management or

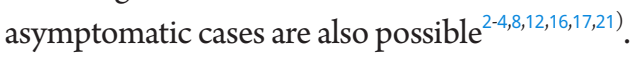

More recently, remote site hemorrhages have been published after successful endovascular treatment of cerebral aneurysms ${ }^{14}$. The suggested mechanisms of this complication have been far from the CSF hypovolemia, and are beyond the scope of the author's discussion.

In summary, it is very crucial for neurosurgeons to remember that 1) most CSF hypovolemias after neurosurgery are probably unnoticed, and thus much underrated. 2) we, neurosurgeons always consider the possibility of above three clinical phenomena for differential diagnosis in case of neurologic deterioration after uneventful neurosurgical procedure. 3) prevention, although limited, of excessive loss of CSF perioperatively may be the best way to treat complications from postoperative CSF hypovolemia. Clear verification of the root pathomechanisms for these unanticipated and tough complications would create further investigations.

\section{CONFLICT OF INTEREST}

No potential conflict of interest relevant to this article was reported.

\section{REFERENCES}

1. Alaraj A, Munson T, Herrera SR, Aletich V, Charbel FT, Amin-Hanjani S. Angiographic features of "brain sag". J Neurosurg 2011;115:586-591.

2. Baek IH, Park KY, Lee JW, Huh SK. Remote cerebellar hemorrhage after surgery for an unruptured aneurysm. Korean J Cerebrovasc Surg 2008;10:454-458.

3. Borkar SA, Lakshmiprasad G, Sharma BS, Mahapatra AK. Remote site intracranial haemorrhage: a clinical series of five patients with review of literature. Br J Neurosurg 2013;27:735-738.

4. Chang SH, Yang SH, Son BC, Lee SW. Cerebellar hemorrhage after burr hole drainage of supratentorial chronic subdural hematoma.J Korean Neurosurg Soc 2009;46:592-595.

5. Francel PC, Persing JA, Cantrell RW, Levine PA, Newman SA. Neurological deterioration after lumbar cerebrospinal fluid drainage. J Craniofac Surg 1992;3:145-148.

6. Guido LJ, Patterson RH Jr. Focal neurological deficits secondary to intraoperative CSF drainage: successful resolution with an epidural blood patch. Report of two cases. J Neurosurg 1976;45:348-351.

7. Kelley GR, Johnson PL. Sinking brain syndrome: craniotomy can precipitate brainstem herniation in CSF hypovolemia. Neurology 2004;62:157.

8. Kim SH, Lee HK, Moon JG, Kim CH, Choi JH. Remote cerebellar hemorrhage after supra- tentorial aneurysm surgery: report of 2 cases. Korean J Cerebrovasc Surg 2008;10:570-574.

9. Komotar RJ, Mocco J, Ransom ER, et al. Herniation secondary to critical postcraniotomy cerebrospinal fluid hypovolemia. Neurosurgery 2005;57:286-292.

10. Komotar RJ, Ransom ER, Mocco J, et al. Critical postcraniotomy cerebrospinal fluid hypovolemia: risk factors and outcome analysis. Neurosurgery 2006;59:284-290.

11.Liu JKC. Neurologic deterioration due to brain sag after bilateral craniotomy for subdural hematoma evacuation. World Neurosurg 2018;114:90-93.

12. Mandonnet E, Faivre B, Bresson D, et al. Supratentorial craniotomy complicated by an homolateral remote cerebellar hemorrhage and a controlateralperisylvian infarction: case report. Acta Neurochir 2010;152:169- 172.

13. Roth J, Galeano E, Milla S, Hartmannsgruber MW, Weiner HL. Multiple Epidural hematomas and hemodynamic collapse caused by a subgaleal drain and suction-induced intracranial hypotension: case report. Neurosurgery 2011;68:E271-E276.

14. Sim SY, Song J, Oh SY, et al. Incidence and characteristics of remote intracerebral hemorrhage after endovascular treatment of unruptured intracranial aneurysms. World Neurosurg 2016;95:335-340.

15. Snow RB, Kuhel W, Martin SB. Prolonged lumbar spinal drainage after the resection of tumors of the skull base: a cautionary note. Neurosurgery 1991;28:880-883.

16. Sturiale CL, Marta Rossetto M, Ermani M, et al. Remote 
cerebellar hemorrhage after supratentorial procedures (part 1): a systematic review. Neurosurg Rev 2016;39:565-573.

17. Sturiale CL, Rossetto M, Ermani M, et al. Remote cerebellar hemorrhage after spinal procedures (part 2): a systematic review. Neurosurg Rev 2016;39:369-376.

18. Tartara F, Bongetta D, Colombo EV, et al. Strokectomy and extensive cerebrospinal fluid drainage for the treatment of space-occupying cerebellar ischemic stroke. World Neurosurg 2018;115:e80-e84.

19. Van Roost D, Thees C, Brenke C, Oppel F, Winkler PA,
Schramm J. Pseudohypoxic brain swelling: a newly defined complication after uneventful brain surgery, probably related to suction drainage. Neurosurgery 2003;53:1315-1327.

20. Yokota H, Yokoyama K, Miyamoto K, Nishioka T. Pseudohypoxic brain swelling after elective clipping of an unruptured anterior communicating artery aneurysm. case report. Clin Neurol Neurosurg 2009; 111:900-903.

21. You SH, Son KR, Lee NJ, Suh JK. Remote cerebral and cerebellar hemorrhage after massive cerebrospinal fluid leakage. J Korean Neurosurg Soc 2012;51:240-243. 